\title{
Family-based whole-exome sequencing identifies novel loss- of-function mutations of FBN1 for Marfan syndrome
}

\author{
Zhening Pu ${ }^{1,2}$, Haoliang Sun ${ }^{3}$, Junjie Du ${ }^{3}$, Yue Cheng ${ }^{1,2}$, Keshuai He ${ }^{3}$, Buqing Ni ${ }^{3}$, Weidong Gu ${ }^{3}$, \\ Juncheng Dai Corresp., 1,2, Yongfeng Shao ${ }^{\text {Corresp. } 3}$ \\ 1 Department of Epidemiology, School of Public Health, Nanjing Medical University, Nanjing, China \\ 2 State Key Laboratory of Reproductive Medicine, Nanjing Medical University, Nanjing, China \\ 3 Department of Cardiovascular Surgery, The First Affiliated Hospital of Nanjing Medical University, Nanjing, China \\ Corresponding Authors: Juncheng Dai, Yongfeng Shao \\ Email address: djc@njmu.edu.cn, yongfengshao30@hotmail.com
}

Background. Marfan syndrome (MFS) is an inherited connective tissue disorder affecting the ocular, skeletal and cardiovascular systems. Previous studies of MFS have demonstrated the association between genetic defects and clinical manifestations. Our purpose was to investigate the role of novel genetic variants in determining MFS clinical phenotypes. Methods. We sequenced the whole exome of 19 individuals derived from three Han Chinese families. The sequencing data were analyzed by a standard pipeline. Variants were further filtered against the public database and an in-house database. Then, we performed pedigree analysis under different inheritance patterns according to American College of Medical Genetics guidelines. Results were confirmed by Sanger sequencing. Results. Two novel loss-of-function indels (c.5027_5028insTGTCCTCC, p.D1677Vfs*8; c.5856delG, p.S1953Lfs*27) and one nonsense variant (c.8034C>A, p.Y2678*) of FBN1 were identified in Family 1, Family 2 and Family 3, respectively. All affected members carried pathogenic mutations, whereas other unaffected family members or control individuals did not. These different kinds of LOF (loss of function) variants of $F B N 1$ were located in the CbEGF region and a conserved domain across species and were not reported previously. Conclusions. Our study extended and strengthened the vital role of FBN1 LOF mutations in the pathogenesis of MFS with autosomal dominant inheritance pattern. We confirm that genetic testing by next-generation sequencing of blood DNA can be fundamental in helping clinicians conduct mutation-based pre- and postnatal screening, genetic diagnosis and clinical management for MFS. 
1 Family-based Whole-exome Sequencing Identifies Novel Loss-of-function Mutations of

2 FBN1 for Marfan syndrome

3 Zhening $\mathrm{Pu}^{1,2}$, Haoliang $\mathrm{Sun}^{3}$, Junjie $\mathrm{Du}^{3}$, Yue Cheng ${ }^{1,2}, \mathrm{Keshuai} \mathrm{He}^{3}$, Buqing $\mathrm{Ni}^{3}$, Weidong $\mathrm{Gu}^{3}$,

4 Juncheng Dai ${ }^{1,2}$, Yongfeng Shao ${ }^{3}$

5

$6{ }^{1}$ Department of Epidemiology, School of Public Health, Nanjing Medical University, Nanjing

$7 \quad 211166$, China

8 2State Key Laboratory of Reproductive Medicine, Nanjing Medical University, Nanjing 211166,

9 China

$10{ }^{3}$ Department of Cardiovascular Surgery, the First Affiliated Hospital of Nanjing Medical

11 University, Nanjing, China

12 Zhening Pu and Haoliang Sun equally contributed to this work.

13 Corresponding author: Yongfeng Shao, yongfengshao30@hotmail.com; Juncheng Dai, Email:

14 djc@njmu.edu.cn 


\section{Abstract}

Background. Marfan syndrome (MFS) is an inherited connective tissue disorder affecting the ocular, skeletal and cardiovascular systems. Previous studies of MFS have demonstrated the association between genetic defects and clinical manifestations. Our purpose was to investigate the role of novel genetic variants in determining MFS clinical phenotypes.

Methods. We sequenced the whole exome of 19 individuals derived from three Han Chinese families. The sequencing data were analyzed by a standard pipeline. Variants were further filtered against the public database and an in-house database. Then, we performed pedigree analysis under different inheritance patterns according to American College of Medical Genetics guidelines. Results were confirmed by Sanger sequencing.

Results. Two novel loss-of-function indels (c.5027_5028insTGTCCTCC, p.D1677Vfs*8; c.5856delG, p.S1953Lfs*27) and one nonsense variant (c.8034C>A, p.Y2678*) of FBN1 were identified in Family 1, Family 2 and Family 3, respectively. All affected members carried pathogenic mutations, whereas other unaffected family members or control individuals did not. These different kinds of LOF (loss of function) variants of FBN1 were located in the cbEGF region and a conserved domain across species and were not reported previously.

Conclusions. Our study extended and strengthened the vital role of FBN1 LOF mutations in the pathogenesis of MFS with autosomal dominant inheritance pattern. We confirm that genetic testing by next-generation sequencing of blood DNA can be fundamental in helping clinicians conduct mutation-based pre- and postnatal screening, genetic diagnosis and clinical management for MFS. 


\section{Introduction}

Marfan syndrome (MFS) is an inherited connective tissue disorder with autosomal dominant transmission. The clinical manifestations of MFS vary from individual to individual. More than 30 different signs and symptoms are variably associated with Marfan syndrome. The most prominent of these affect the skeletal, cardiovascular, and ocular systems, but all fibrous connective tissue throughout the body can be affected (Pyeritz \& McKusick 1979). Clinically, aortic dilatation and dissection are the most important and life-threatening manifestations of MFS (Biggin et al. 2004). The estimated prevalence is 1 in 5,000 individuals, without gender predilection (Sponseller et al. 1995; von Kodolitsch \& Robinson 2007). An epidemiological study in Taiwan revealed that the overall prevalence of MFS in Chinese population was $10.2(95 \% \mathrm{CI}$, 9.8-10.7) per 100,000 individuals (Chiu et al. 2014).

Mutations in FBN1 (OMIM \#134797, encoding fibrillin-1) account for $70 \%-80 \%$ of MFS (Stheneur et al. 2009). In addition to FBN1, there are other candidate genes functionally related to MFS, such as TGFBR1, TGFBR2, ACTA2, SMAD3, MYH11 and MYLK. Habashi et al. showed that aortic aneurysm in a mouse model of MFS is associated with increased TGF-beta signaling (Habashi et al. 2006) and noncanonical (Smad-independent) TGF-beta signaling may be a prominent driver of aortic disease in Marfan syndrome mice (Holm et al. 2011). Traditionally, the discovery of pathogenic genes for MFS has depended on locus mapping using a candidate-gene strategy with family-based designs, while FBN1 mutations have not been detected in $10 \%$ of MFS patients from clinical diagnosis, implying that either atypical mutation types or 60 other genes may cause MFS-like disease ( $\mathrm{Li}$ et al. 2017). Most cases inherit MFS from their parents 
61 in an autosomal dominant fashion (Wieczorek et al. 1996). Marfan syndrome may also be caused

62 by dominant negative-typemutations and haploinsufficiency (Hilhorst-Hofstee et al. 2011; Judge

63 et al. 2004; Judge \& Dietz 2005). Therefore, more pathogenic genes or atypical mutations in

64 specific populations remain to be identified. Here, we performed a family-based study using

65 whole-exome sequencing (WES) in 19 individuals, who were derived from three Han Chinese

66 MFS families. We identified three novel LOF mutations in FBN1 likely to cause MFS in these

67 patients. Systematical evaluations and experimental replications were conducted to validate our

68 findings.

69

70

71

72

73

74

\section{Materials and Methods}

\section{Study Subjects}

A total of 19 volunteers from three Han Chinese families were recruited from the First Affiliated Hospital of Nanjing Medical University between 2012 and 2016. The mean age of onset of cases was 24.6 \pm 6.8 years (Family 1: I-1 lost to follow-up). MFS was diagnosed through a medical record review, physical examination, and family history based on Ghent nosology: (i) Ectopia lentis; (ii) Systemic score $\geq 7$; (iii) Aortic root Z-score $\geq 2$, when there is history of Marfan syndrome in a primary relative (Loeys et al. 2010). The study was approved by the institutional ethical committee of Nanjing Medical University and complied with the principles of the declaration of Helsinki. Informed consent was obtained from all subjects.

\section{Whole-exome sequencing}

Genomic DNA was isolated from peripheral blood using the QIAamp ${ }^{\mathrm{TM}}$ DNA and Blood Mini kit $\left(\right.$ Qiagen $\left.^{\mathrm{TM}}\right)$ according to the protocol. Total DNA concentration and quantity were assessed by measuring absorbance at $260 \mathrm{~nm}$ with NanoDrop 2000c Spectrophotometer (Thermo Scientific ${ }^{\mathrm{TM}}$ ). 
84 WES library construction and sequencing were performed as below: 300ng genomic DNA was

85 fragmented in a Covaris ${ }^{\circledR}$ M220 Focused-ultrasonicator ${ }^{\mathrm{TM}}$ to $100-500 \mathrm{bp}$ fragments followed by

86 end repair, adding A-tailing, adaptor ligation, and 11 PCR cycles according to the manufacturer's

87 protocols. After hybridization, exome enrichment was conducted with the Agilent XT SureSelect

Human All Exon v5 Kit, which targets $\sim 50 \mathrm{Mb}$ of the human exonic regions. Five DNA libraries

89

91

92

93 were multiplexed on every lane and 101 base paired-end sequencing was performed on Illumina HiSeq1500 (Illumina, Inc).

\section{Quality control, mapping and variant calling}

Raw sequencing reads were filtered to trim adapters and low quality reads using Trimmomatic0.3.2 under PE module (ILLUMINACLIP: adapter. fa: 2:30:10; LEADING: 3; TRAILING: 3; SLIDINGWINDOW: 4:15; MINLEN: 20). All the qualified reads were processed with an in-house bioinformatics pipeline, which followed the best practice steps suggested by Genome Analysis Toolkit (GATK v3.5) (DePristo et al. 2011). Briefly, we first aligned the clean sequence reads to the human reference genome (UCSC Genome Browser hg19) using Burrows-Wheeler Aligner (BWA-MEM v0.7.12 with default parameters) (Li \& Durbin 2010). PCR duplicates were removed by Picard v1.141. After initial quality control (QC), all eligible sequences were determined for regional realignment and base quality recalibration with GATK v3.5. Then variations including single nucleotide variants (SNVs), insertions and deletions were called using GATK v3.5 HaplotypeCaller module.

\section{Gene Classification and Functional annotation}

We used Annovar (2016Feb01) (Wang et al. 2010) for functional annotation with Online 
107 Mendelian Inheritance in Man (OMIM), the Exome Aggregation Consortium (ExAC) Browser, 108 MutationTaster2 and The Combined Annotation Dependent Depletion (CADD). Based on OMIM 109 and MFS-related literature reported previously, genes were classified into three categories 110 according to American College of Medical Genetics (ACMG) standards and guidelines (Richards

111 et al. 2015): Category I: 8 MFS-causing genes reported directly; Category II: 125 MFS-related 112 genes from GeneCards; Category III: Unknown genes not reported previously (Supplementary 113 Table 1).

114

115

116

117

118

119

120

121

122

123

124

125

126

127

128

129

\section{Manual review and replication using Sanger sequencing}

All remaining mutations were manually inspected using the Integrated Genome Viewer (IGV 2.3.80) (Thorvaldsdottir et al. 2013) before Sanger sequencing. PCR primers were designed for the target regions and were used to amplify these regions by PCR for Sanger sequencing. Primers are listed in the Supplementary Table 2. Mutations were validated according to the resulting data screened through Chromas 2.4.1 and Dnaman 6.0.

\section{Results}

One 18-year-old male (the proband, II-1, age of onset was 10) of Han Chinese ancestry from Family 1 was diagnosed with MFS. He presented with acute, anterior chest pain, on admission. His father (I-1) also had MFS (Fig. 1A). Computed Tomography (CT) revealed that the proband had an aortic aneurysm with dissection (type III), ascending aortic root dilatation with the diameter of $4.9 \mathrm{~cm}$. Family 2 is a large three-generation family with five family members affected (I-2, I-5, I-6, II-1 and III-1) (Fig. 1B). The proband (II-1, age of onset was 30), a 31-year-old male, presented with elongated digits but no pectus excavatum. CT showed an aortic aneurysm with dissection 
130 (type I). His mother (I-2), one aunt (I-5) and one uncle (I-6) all had MFS with aortic aneurysms. 131 His mother underwent surgery for aortic dissection in 1993 and died in 2015. Family 3 spanned 132 three generations with two family members affected (I-2 and II-2). The proband (II-2, age of onset was 27) was a 28-year-old male with a history of MFS. He had thoracic surgery for pectus excavatum at 2 years old. Although he had no clinical symptoms in the cardiovascular system, a CT scan showed ascending aortic dilatation, aortic regurgitation and mitral regurgitation. His mother (I-2) was also confirmed to have MFS (Fig. 1C). Although case I-3 presented elongation of fingers and mild pectus excavatum, there were not sufficient clinical features to perform the diagnosis (

Quality summaries from sequencing of the 19 samples is summarized in Supplementary Table 3. Each sample had an average of $69.46 \mathrm{M}$ raw reads, and over $99.60 \%$ of them were successfully mapped to the reference genome. The average of median insert size was $201 \mathrm{bp}$ and percent of duplicate reads ranged from $1.44 \%$ to $8.11 \%$. Totally 237,252 variants were kept for following evaluation. To identify qualified pathogenic mutations, stringent criteria according to ACMG guidelines were performed (Supplementary Fig. 1). First, we filtered these variants under the following criteria: (i) untranslated region, synonymous, intronic variants (except variants considered to be splicing variants and located at exon-intron junctions ranging from -5 to +5 ); (ii) variants with minor allele frequency $(\mathrm{MAF}) \geq 1 \%$ based on 1000 Genomes $(1 \mathrm{KG})$ and ExAC databases; (iii) variants present in our in-house whole genome sequencing (WGS) database of 100 non-Marfan controls. Then, we classified these rare genetic variants into three categories: a. MFScausing genes; b. MFS-related genes; c. Unknown genes. Then we assessed whether these variants were loss-of-function (nonsense, frameshift and essential splice-site variants). Three inheritance patterns were evaluated for the qualified mutations including autosomal dominant, autosomal 
153 recessive and compound heterozygotes. Finally, we manually reviewed and selected variants in

154 Category I and II genes for validation. After replication by Sanger sequencing, three LOF

155 mutations in FBN1 were identified in three families respectively. For Family 1, the insertion

156 (c.5027_5028insTGTCCTCC) was detected in both I-1 and II-1 individuals, which resulted in a

157 frameshift (p.D1677Vfs*8). For Family 2, a heterozygous deletion (c.5856delG) in exon 48

158 (NM_000138) was found in four patients (I-5, I-6, II-1 and III-1), also resulting in a frameshift

159 (p.S1953Lfs*27). I-2 and II-2 individuals from family 3 were heterozygous for the nonsense

160 variant (c.8034C $>$ A), which gained an immediate stop codon (p.Y2678*). All mutations

161 (c.5027_5028insTGTCCTCC, c.5856delG and c.8034C>A) identified in three families were

162 predicted to be disease-causing using MutationTaster2 and CADD. In addition, all mutation sites

163 were located in a highly conserved amino acid region (Calcium-binding EGF domain) across

164 different species (Supplementary Fig. 2). A summary of these mutations is presented in Table 2.

165 All healthy family members and 100 other non-MFS controls did not carry these FBN1 variants.

166 It is worth noting that these mutations in FBN1 have not been reported previously, but the variant

167 (c.5857dupT), near c.5856delG, was recorded by the Human Gene Mutation Database (HGMD).

\section{Discussion}

170 MFS is a systemic disorder of connective tissue with a high degree of clinical variability that involves skeletal, ocular, and cardiovascular systems (Dietz 1993). In our study, massively parallel sequencing was performed to identify genetic abnormalities in three MFS families, showing three rare functional variations in $F B N 1$.

Fibrillins are the major components of microfibrils in the extracellular matrix of elastic and non175 elastic tissues. Fibrillin-1 serves as a structural component of calcium-binding microfibrils and is 
176

177

178

179

180

181

182

183

184

185

186

187

188

189

190

191

192

193

194

195

196

197

198

encoded by FBN1 gene. FBN1 is mapped to chromosome 15q21.1 and encodes a 2,871 amino acid protein. More than 1,800 different mutations have been identified in $F B N 1$, most of which are associated with MFS, as seen in the UMD-FBN1 mutations database (Collod-Beroud et al. 1997; Collod et al. 1996) using a generic software called Universal Mutation Database (UMD) (CollodBeroud et al. 2003). Similar to this curated database, approximately 1,700 variants in FBN1 are associated with MFS according to the professional version of The Human Gene Mutation Database (HGMD), and these accounts for more than $90 \%$ of MFS cases. In our study, all affected individuals also carried FBN1 LOF mutations (c.5027_5028insTGTCCTCC, c.5856delG and c. $8034 \mathrm{C}>\mathrm{A})$.

The mutated mRNAs in our study, although introducing premature termination codons (PTC), could in theory be stable. However, cellular recognition and degradation of mRNA that contains PTC via nonsense-mediated mRNA decay (NMD) is a process whereby potentially harmful effects of truncated proteins may be limited (Culbertson 1999; Frischmeyer \& Dietz 1999). Accordingly to one study, in the majority of FBN1 PTC (premature termination codon) MFS cases, synthesis of normal-sized fibrillin protein was $\sim 50 \%$ of control levels, but much less matrix deposition occurred (Schrijver et al. 2002). They concluded that most PTC mutations have a major impact on the pathogenesis of type 1 fibrillinopathies but that it is not always through NMD. In our study, we found that two PTC mutations (p.S1953Lfs*27 and p.Y2678*) were quite near the mutations (p.Q1955X and p.I2681X) reported by Schrijver et al (Schrijver et al. 2002). In contrast, the relative amount of PTC-containing FBN1 transcripts in blood was found to be significantly higher than in affected fibroblasts with experimental inhibition of nonsense-mediated decay, while in fibroblasts without NMD inhibition, no mutant alleles could be detected at all (Magyar et al. 2009), implying that tissue-specific degradation of transcripts also plays an important role in MFS 
199

200

201

202

203

204

205

206

207

208

209

210

211

212

213

214

215

216

217

218

219

220

221

pathogenesis.

Along these lines, Faivre et al. found that patients with an FBN1 PTC had a more severe skeletal and skin phenotype than did patients with an in-frame mutation. Mutations in exons 24-32 were also associated with a more severe and complete phenotype (Faivre et al. 2007). In our study, the LOF mutations were located in exon 41, 48 and 64, and patients did not have a complete MFS phenotype including ectopia lentis and skin striae. This expression of the MFS phenotype may also depend on the different ethnicity of our patient cohort from the above-cited studies.

In family 2, a suspected case of a 4-year-old boy (patient III-1) who had longer finger and anterior chest deformity (pectus excavatum), was slightly taller than his peers. It was difficult to make a clinical diagnosis owing to his age and uncertain status according to the clinical criteria, but our WES-based screening helped ease his diagnosis by excluding the FBN1 mutation found in other affected members of his family. In family 3, we detected that clinically unaffected subject I-3 had a slight anterior chest deformity (pectus excavatum). Although she had this MFS-related symptom, she was clinically considered as a healthy individual, which was borne out by the result that she had no FBN1 or other pathogenic mutations.

All family members above are followed up regularly to confirm their diagnoses. The identification of a causative gene variant by WES in those with an uncertain phenotype or complex subjects is of inestimable value for screening, clinical diagnosis and, ultimately, directing personalized patient care with development of specific small-molecule therapies.

\section{Conclusions}

In conclusion, our results may help further elucidate the genetic pathology of MFS, and these mutations could be included among probably pathogenic markers for pre- and postnatal screening 
222 and genetic diagnosis for MFS. 


\section{Web Resources}

224 Qiagen ${ }^{\mathrm{TM}}$, https://www.qiagen.com

225 Agilent ${ }^{\mathrm{TM}}$, https://www.agilent.com

226 Trimmomatic-0.3.2, http://www.usadellab.org/cms/index.php?page=trimmomatic

227 Genome Analysis Toolkit (GATK v3.5), https://software.broadinstitute.org/gatk

228 Burrows-Wheeler Aligner, BWA v0.7.12, http://bio-bwa.sourceforge.net

229 Picard v1.141, http://picard.sourceforge.net

230 Annovar (2016Feb01), http://annovar.openbioinformatics.org

231 Mendelian Inheritance in Man (OMIM), http://www.omim.org

232 Exome Aggregation Consortium (ExAC) Browser, http://exac.broadinstitute.org

233 MutationTaster2, http://www.mutationtaster.org/

234 The Combined Annotation Dependent Depletion (CADD), http://cadd.gs.washington.edu

235 GeneCards, https://www.genecards.org/Search/Keyword?queryString=marfan\%20syndrom

236 Integrated Genome Viewer (IGV 2.3.80), http://software.broadinstitute.org/software/igv/

237 Human Gene Mutation Database (HGMD), http://www.hgmd.cf.ac.uk

238 Universal Mutation Database (UMD), http://www.umd 
240

241

242

243

244

245

246

247

248

249

250

251

252

253

254

255

256

257

258

259

260

261

262

263

264

265

266

267

268

269

270

271

272

273

274

275

276

277

278

279

280

281

282

283

284

285

286

287

288

289

290

\section{References}

Biggin A, Holman K, Brett M, Bennetts B, and Ades L. 2004. Detection of thirty novel FBN1 mutations in patients with Marfan syndrome or a related fibrillinopathy. Hum Mutat 23:99. 10.1002/humu.9207

Chiu HH, Wu MH, Chen HC, Kao FY, and Huang SK. 2014. Epidemiological profile of Marfan syndrome in a general population: a national database study. Mayo Clin Proc 89:34-42. 10.1016/j.mayocp.2013.08.022

Collod-Beroud G, Beroud C, Ades L, Black C, Boxer M, Brock DJ, Godfrey M, Hayward C, Karttunen L, Milewicz D, Peltonen L, Richards RI, Wang M, Junien C, and Boileau C. 1997. Marfan Database (second edition): software and database for the analysis of mutations in the human FBN1 gene. Nucleic Acids Res 25:147-150.

Collod-Beroud G, Le Bourdelles S, Ades L, Ala-Kokko L, Booms P, Boxer M, Child A, Comeglio P, De Paepe A, Hyland JC, Holman K, Kaitila I, Loeys B, Matyas G, Nuytinck L, Peltonen L, Rantamaki T, Robinson P, Steinmann B, Junien C, Beroud C, and Boileau C. 2003. Update of the UMD-FBN1 mutation database and creation of an FBN1 polymorphism database. Hum Mutat 22:199-208. 10.1002/humu.10249

Collod G, Beroud C, Soussi T, Junien C, and Boileau C. 1996. Software and database for the analysis of mutations in the human FBN1 gene. Nucleic Acids Res 24:137-140.

Culbertson MR. 1999. RNA surveillance. Unforeseen consequences for gene expression, inherited genetic disorders and cancer. Trends Genet 15:74-80.

DePristo MA, Banks E, Poplin R, Garimella KV, Maguire JR, Hartl C, Philippakis AA, del Angel G, Rivas MA, Hanna M, McKenna A, Fennell TJ, Kernytsky AM, Sivachenko AY, Cibulskis K, Gabriel SB, Altshuler D, and Daly MJ. 2011. A framework for variation discovery and genotyping using next-generation DNA sequencing data. Nat Genet 43:491-498. 10.1038/ng.806

Dietz H. 1993. Marfan Syndrome. In: Adam MP, Ardinger HH, Pagon RA, Wallace SE, Bean LH, Mefford HC, Stephens $\mathrm{K}$, Amemiya A, and Ledbetter N, eds. GeneReviews((R)). Seattle (WA).

Faivre L, Collod-Beroud G, Loeys BL, Child A, Binquet C, Gautier E, Callewaert B, Arbustini E, Mayer K, Arslan-Kirchner M, Kiotsekoglou A, Comeglio P, Marziliano N, Dietz HC, Halliday D, Beroud C, Bonithon-Kopp C, Claustres M, Muti C, Plauchu H, Robinson PN, Ades LC, Biggin A, Benetts B, Brett M, Holman KJ, De Backer J, Coucke P, Francke U, De Paepe A, Jondeau G, and Boileau C. 2007. Effect of mutation type and location on clinical outcome in 1,013 probands with Marfan syndrome or related phenotypes and FBN1 mutations: an international study. Am J Hum Genet 81:454-466. 10.1086/520125

Frischmeyer PA, and Dietz HC. 1999. Nonsense-mediated mRNA decay in health and disease. Hum Mol Genet 8:18931900.

Habashi JP, Judge DP, Holm TM, Cohn RD, Loeys BL, Cooper TK, Myers L, Klein EC, Liu G, Calvi C, Podowski M, Neptune ER, Halushka MK, Bedja D, Gabrielson K, Rifkin DB, Carta L, Ramirez F, Huso DL, and Dietz HC. 2006. Losartan, an AT1 antagonist, prevents aortic aneurysm in a mouse model of Marfan syndrome. Science 312:117-121. 10.1126/science.1124287

Hilhorst-Hofstee Y, Hamel BC, Verheij JB, Rijlaarsdam ME, Mancini GM, Cobben JM, Giroth C, Ruivenkamp CA, Hansson KB, Timmermans J, Moll HA, Breuning MH, and Pals G. 2011. The clinical spectrum of complete FBN1 allele deletions. Eur J Hum Genet 19:247-252. 10.1038/ejhg.2010.174

Holm TM, Habashi JP, Doyle JJ, Bedja D, Chen Y, van Erp C, Lindsay ME, Kim D, Schoenhoff F, Cohn RD, Loeys BL, Thomas CJ, Patnaik S, Marugan JJ, Judge DP, and Dietz HC. 2011. Noncanonical TGFbeta signaling contributes to aortic aneurysm progression in Marfan syndrome mice. Science 332:358-361. 10.1126/science.1192149

Judge DP, Biery NJ, Keene DR, Geubtner J, Myers L, Huso DL, Sakai LY, and Dietz HC. 2004. Evidence for a critical contribution of haploinsufficiency in the complex pathogenesis of Marfan syndrome. J Clin Invest 114:172181. $10.1172 / \mathrm{JCl} 20641$

Judge DP, and Dietz HC. 2005. Marfan's syndrome. Lancet 366:1965-1976. 10.1016/S0140-6736(05)67789-6

$\mathrm{Li} \mathrm{H}$, and Durbin R. 2010. Fast and accurate long-read alignment with Burrows-Wheeler transform. Bioinformatics 26:589-595. 10.1093/bioinformatics/btp698

Li J, Wu W, Lu C, Liu Y, Wang R, Si N, Liu F, Zhou J, Zhang S, and Zhang X. 2017. Gross deletions in FBN1 results in variable phenotypes of Marfan syndrome. Clin Chim Acta 474:54-59. 10.1016/j.cca.2017.08.023

Loeys BL, Dietz HC, Braverman AC, Callewaert BL, De Backer J, Devereux RB, Hilhorst-Hofstee Y, Jondeau G, Faivre L, Milewicz DM, Pyeritz RE, Sponseller PD, Wordsworth P, and De Paepe AM. 2010. The revised Ghent 
nosology for the Marfan syndrome. J Med Genet 47:476-485. 10.1136/jmg.2009.072785

Magyar I, Colman D, Arnold E, Baumgartner D, Bottani A, Fokstuen S, Addor MC, Berger W, Carrel T, Steinmann B, and Matyas G. 2009. Quantitative sequence analysis of FBN1 premature termination codons provides evidence for incomplete NMD in leukocytes. Hum Mutat 30:1355-1364. 10.1002/humu.21058

Pyeritz RE, and McKusick VA. 1979. The Marfan syndrome: diagnosis and management. N Engl J Med 300:772-777. 10.1056/NEJM197904053001406

Richards S, Aziz N, Bale S, Bick D, Das S, Gastier-Foster J, Grody WW, Hegde M, Lyon E, Spector E, Voelkerding K, Rehm HL, and Committee ALQA. 2015. Standards and guidelines for the interpretation of sequence variants: a joint consensus recommendation of the American College of Medical Genetics and Genomics and the Association for Molecular Pathology. Genet Med 17:405-424. 10.1038/gim.2015.30

Schrijver I, Liu W, Odom R, Brenn T, Oefner P, Furthmayr H, and Francke U. 2002. Premature termination mutations in FBN1: distinct effects on differential allelic expression and on protein and clinical phenotypes. Am J Hum Genet 71:223-237. 10.1086/341581

Sponseller PD, Hobbs W, Riley LH, 3rd, and Pyeritz RE. 1995. The thoracolumbar spine in Marfan syndrome. J Bone Joint Surg Am 77:867-876.

Stheneur C, Collod-Beroud G, Faivre L, Buyck JF, Gouya L, Le Parc JM, Moura B, Muti C, Grandchamp B, Sultan G, Claustres M, Aegerter P, Chevallier B, Jondeau G, and Boileau C. 2009. Identification of the minimal combination of clinical features in probands for efficient mutation detection in the FBN1 gene. Eur J Hum Genet 17:1121-1128. 10.1038/ejhg.2009.36

Thorvaldsdottir H, Robinson JT, and Mesirov JP. 2013. Integrative Genomics Viewer (IGV): high-performance genomics data visualization and exploration. Brief Bioinform 14:178-192. 10.1093/bib/bbs017

von Kodolitsch Y, and Robinson PN. 2007. Marfan syndrome: an update of genetics, medical and surgical management. Heart 93:755-760. 10.1136/hrt.2006.098798

Wang K, Li M, and Hakonarson H. 2010. ANNOVAR: functional annotation of genetic variants from high-throughput sequencing data. Nucleic Acids Res 38:e164. 10.1093/nar/gkq603

Wieczorek P, Riegel MB, Quattro L, and DeMaio K. 1996. Marfan's syndrome and surgical repair of ascending aortic aneurysms. AORN J 64:895-913; quiz 916-898, 921-892.

\section{Abbreviations:}

Figure 1. Pedigree and mutations in FBN1 for Marfan syndrome patients

1A: A FBN1 insertion mutation (M1) was identified in two subjects with MFS (I-1 and II-1) from

Family 1; 1B: A FBN1 deletion mutation (M2) was identified in four subjects with MFS (I-5, I-6, II-1 and III-1) from Family 2; 1C: A FBN1 nonsense mutation (M3) was identified in two subjects with MFS (I-2 and II-2) from Family 3. 3 individuals in the pedigrees were not sequenced including Family 2: I-2, I-7 and Family 3: I-4. W indicates wildtype allele. Circles represent female participants and squares male participants. Black symbols indicate patients with Marfan syndrome. A slash through the symbol indicates that the family member is deceased. Arrows indicate the proband. 
331 Supplementary Figure 1. Analytical pipeline for exome sequence filtration and

332 prioritization

333 Abbreviations: MAF, minor allele frequency; UTR, untranslated region.

334

335 Supplementary Figure 2 The locations of mutations in FBN1 across different species

336 The positions of three LOF mutations in FBN1 are shown. The affected amino acid residues are 337 conserved across multiple species. 


\section{Table $\mathbf{1}$ (on next page)}

Clinical symptoms of all 19 members in three Marfan families

Facial features $(3 / 5)=1$ (dolichocephaly, enophthalmos, downslanting palpebral fissures, malar hypoplasia, retrognathia) ND $=$ not detected $N A=$ not available $*$ Suspected case 
1 Table 1. Clinical symptoms of all 19 members in three Marfan families

\begin{tabular}{|c|c|c|c|c|c|c|c|c|c|c|c|c|c|c|c|c|c|c|c|}
\hline $\begin{array}{c}\text { Family } \\
\text { ID }\end{array}$ & $\begin{array}{c}\text { Member } \\
\text { ID }\end{array}$ & $\begin{array}{l}\text { Age of } \\
\text { onset }\end{array}$ & Age & $\begin{array}{c}\text { Wrist } \\
\text { AND } \\
\text { thumb } \\
\text { sign }\end{array}$ & $\begin{array}{l}\text { Pectus carinatum } \\
\text { deformity(pectus } \\
\text { excavatum or chest } \\
\text { asymmetry) }\end{array}$ & $\begin{array}{c}\text { Hindfoot } \\
\text { deformity } \\
\text { (plain pes } \\
\text { planus) }\end{array}$ & $\begin{array}{l}\text { Dural } \\
\text { ectasia }\end{array}$ & $\begin{array}{l}\text { Protrusio } \\
\text { acetabuli }\end{array}$ & Pneumothorax & $\begin{array}{c}\text { Reduced upper } \\
\text { segment/lower } \\
\text { segment ratio } \\
\text { AND increased } \\
\text { arm/height AND } \\
\text { no severe } \\
\text { scoliosis }\end{array}$ & $\begin{array}{c}\text { Scoliosis or } \\
\text { thoracolumbar } \\
\text { kyphosis } \\
\end{array}$ & $\begin{array}{l}\text { Reduced } \\
\text { elbow } \\
\text { extension }\end{array}$ & $\begin{array}{l}\text { Facial } \\
\text { features }\end{array}$ & $\begin{array}{c}\text { Skin } \\
\text { striae } \\
\text { (stretch } \\
\text { marks) }\end{array}$ & $\begin{array}{c}\text { Myopia > } \\
3 \\
\text { diopters }\end{array}$ & $\begin{array}{l}\text { Mitral } \\
\text { valve } \\
\text { prolapse }\end{array}$ & $\begin{array}{c}\text { Systemic } \\
\text { score }\end{array}$ & $\begin{array}{l}\text { Aortic } \\
\text { root Z- } \\
\text { score }\end{array}$ & Case \\
\hline Score & & & & 3 & $2(1)$ & $2(1)$ & 2 & 2 & 2 & 1 & 1 & 1 & 1 & 1 & 1 & 1 & & & \\
\hline F1 & I-1 & 25 & 46 & $\sqrt{ }$ & $\sqrt{ }$ & $\times$ & $\times$ & $x$ & $\sqrt{ }$ & x & $\times$ & $x$ & $\times$ & $\times$ & $\times$ & $\sqrt{ }$ & 8 & ND & 1 \\
\hline F1 & I-2 & & 43 & $\times$ & $x$ & $\times$ & $\times$ & $x$ & $\times$ & $\times$ & $\times$ & $\times$ & $x$ & $\times$ & $\times$ & $\times$ & 0 & ND & 0 \\
\hline F1 & II-1 & 10 & 21 & $\sqrt{ }$ & $\sqrt{ }$ & $\times$ & $x$ & x & $\sqrt{ }$ & $\times$ & $\times$ & $\times$ & $\times$ & x & $\times$ & $\sqrt{ }$ & 8 & $\geq 2$ & 1 \\
\hline F2 & I-1 & & 66 & $\times$ & $\times$ & $\times$ & $\times$ & $x$ & $x$ & $\times$ & $\times$ & $x$ & $x$ & $\times$ & $\times$ & $\times$ & 0 & ND & 0 \\
\hline F2 & I-3 & & 60 & $\times$ & $\times$ & $\times$ & $\times$ & $\times$ & $x$ & $\times$ & $\times$ & $x$ & $\times$ & $\times$ & $\times$ & $\times$ & 0 & ND & 0 \\
\hline F2 & I-4 & & 63 & $\times$ & $\times$ & $\times$ & $\times$ & $\times$ & $\times$ & $\times$ & $\times$ & $\times$ & $\times$ & $x$ & $\times$ & $\times$ & 0 & ND & 0 \\
\hline F2 & I-5 & 30 & 65 & x & $\times$ & $\sqrt{ }$ & $\times$ & x & $x$ & $\checkmark$ & $\times$ & $\sqrt{ }$ & $\sqrt{ }$ & $x$ & $\times$ & $\sqrt{ }$ & 6 & ND & 1 \\
\hline F2 & I-6 & 20 & 67 & $x$ & $x$ & $\sqrt{ }$ & $x$ & $x$ & $x$ & $\sqrt{ }$ & $x$ & $\sqrt{ }$ & $\sqrt{ }$ & x & $\times$ & $\sqrt{ }$ & 6 & $\mathrm{ND}$ & 1 \\
\hline
\end{tabular}




\begin{tabular}{|c|c|c|c|c|c|c|c|c|c|c|c|c|c|c|c|c|c|c|c|}
\hline F2 & II-1 & 30 & 36 & $x$ & $x$ & $\sqrt{ }$ & $\times$ & $x$ & $\times$ & $\sqrt{ }$ & $x$ & $\sqrt{ }$ & $\sqrt{ }$ & $x$ & $\times$ & $\sqrt{ }$ & 6 & $\geq 2$ & 1 \\
\hline F2 & II-2 & & 30 & $x$ & $\times$ & $x$ & $\times$ & $x$ & $\times$ & $x$ & $x$ & $\times$ & $\times$ & $x$ & $\times$ & $\times$ & 0 & ND & 0 \\
\hline F2 & II-3 & & 50 & $x$ & $x$ & $x$ & $\times$ & $x$ & $\times$ & $\times$ & $x$ & $\times$ & $\times$ & $x$ & $\times$ & $\times$ & 0 & ND & 0 \\
\hline F2 & III-1* & 2 & 5 & $x$ & $\sqrt{ }$ & $x$ & $x$ & $x$ & $x$ & $x$ & $x$ & $x$ & $x$ & $x$ & $\times$ & $\times$ & 1 & $\mathrm{ND}$ & $0^{*}$ \\
\hline F3 & I-1 & & 52 & $x$ & $x$ & $x$ & $\times$ & $x$ & $x$ & $x$ & $x$ & $\times$ & $\times$ & $x$ & $\times$ & $x$ & 0 & ND & 0 \\
\hline F3 & I-2 & 30 & 49 & $\times$ & $\sqrt{ }$ & $\sqrt{ }$ & $\times$ & $\times$ & $\times$ & $x$ & $\times$ & $\times$ & $\times$ & $x$ & $\times$ & $\sqrt{ }$ & 5 & ND & 1 \\
\hline F3 & I-3 & & 46 & $\sqrt{ }$ & $\sqrt{ }$ & $x$ & $\times$ & $x$ & $x$ & $\times$ & $x$ & $\times$ & $\times$ & $x$ & $\times$ & $\times$ & 4 & $<2$ & 0 \\
\hline F3 & II-2 & 27 & 29 & $x$ & $\sqrt{ }$ & $\sqrt{ }$ & $\times$ & $\times$ & $x$ & $\times$ & $x$ & $\times$ & $\times$ & $x$ & $\times$ & $\sqrt{ }$ & 5 & $\geq 2$ & 1 \\
\hline F3 & II-3 & & 28 & $x$ & $x$ & $x$ & $x$ & $x$ & $x$ & $\times$ & $x$ & $x$ & $x$ & $x$ & $\times$ & $\times$ & 0 & ND & 0 \\
\hline F3 & II-4 & & 22 & $x$ & $\times$ & x & $x$ & $x$ & $x$ & $\times$ & $\times$ & $\times$ & $\times$ & $x$ & $\times$ & $\times$ & 0 & $\mathrm{ND}$ & 0 \\
\hline F3 & III-1 & & 6 & $x$ & $\times$ & $x$ & $x$ & $x$ & $x$ & $x$ & $x$ & $x$ & $x$ & $x$ & $x$ & $x$ & 0 & ND & 0 \\
\hline
\end{tabular}

2 Facial features $(3 / 5)=1$ (dolichocephaly, enophthalmos, downslanting palpebral fissures, malar hypoplasia, retrognathia)

$3 \mathrm{ND}=$ not detected

$4 \quad \mathrm{NA}=$ not available

$5 \quad *$ Suspected case 
Table 2 (on next page)

FBN1 variants identified for affected individuals in three Marfan families 
1 Table 2. FBN1 variants identified for affected individuals in three Marfan families

\begin{tabular}{|c|c|c|c|}
\hline Family ID & F1 & F2 & F3 \\
\hline Chr. & $\operatorname{chr} 15$ & $\operatorname{chr} 15$ & $\operatorname{chr} 15$ \\
\hline Position & $48,756,133$ & $48,737,634$ & $48,707,750$ \\
\hline Ref allele & - & G & $\mathrm{C}$ \\
\hline Alt allele & TGTCCTCC & - & A \\
\hline Gene & FBN1 & FBN1 & FBN1 \\
\hline Mutation type & insertion & deletion & nonsense \\
\hline Exon & $41 / 66$ & $48 / 66$ & $64 / 66$ \\
\hline Codon change & c.5027_5028insTGTCCTCC & c.5856delG & c. $8034 \mathrm{C}>\mathrm{A}$ \\
\hline Amino acid change & p.D1677Vfs*8 & p.S1953Lfs*27 & p.Y2678* \\
\hline Affected individuals & I-1/II-1 & I-5/I-6/II-1/III-1 & $\mathrm{I}-2 / \mathrm{II}-2$ \\
\hline CADD Raw Score & 9.18 & 7 & 16.63 \\
\hline PHRED scaled score ${ }^{\dagger}$ & 35 & 33 & 56 \\
\hline
\end{tabular}

${ }^{\dagger}$ PHRED-like scaled C-scores $=-10 * \log _{10}\left(\frac{\text { rank }}{\text { total }}\right)$, the recommended deleterious threshold was $>15$ for scaled C-scores 


\section{Figure 1}

Pedigree and mutations in FBN1 for Marfan syndrome patients

1A: A FBN1 insertion mutation (M1) was identified in two subjects with MFS (I-1 and II-1) from Family 1; 1B: A FBN1 deletion mutation (M2) was identified in four subjects with MFS (I-5, I-6, II-1 and III-1) from Family 2; 1C: A FBN1 nonsense mutation (M3) was identified in two subjects with MFS (I-2 and II-2) from Family 3. 3 individuals in the pedigrees were not sequenced including Family 2: I-2, I-7 and Family 3: I-4. W indicates wildtype allele. Circles represent female participants and squares male participants. Black symbols indicate patients with Marfan syndrome. A slash through the symbol indicates that the family member is deceased. Arrows indicate the proband.

A. Family 1

M1=c.5027_5028insTGTCCTCC, p.D1677Vfs*8

I

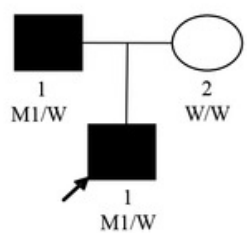

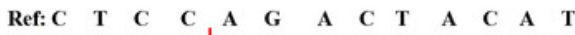

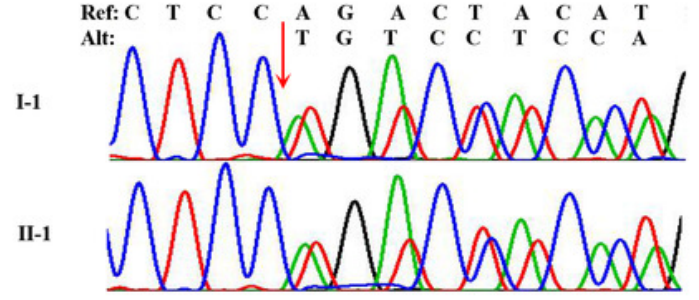

C. Family 3

$\mathrm{M} 3=\mathrm{c} .8034 \mathrm{C}>\mathrm{A}, \mathrm{p} . \mathrm{Y} 2678^{*}$

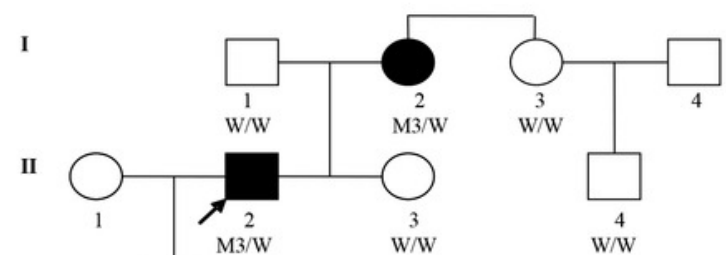

III
B. Family 2

M2=c.5856delG, p.S1953Lfs*27

I

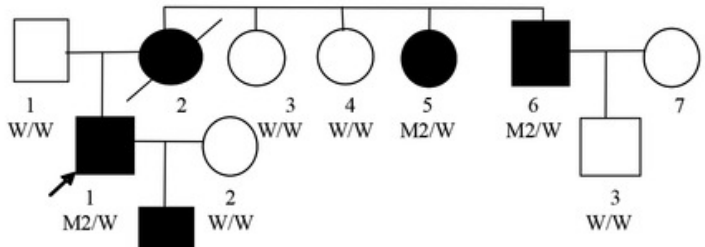

III

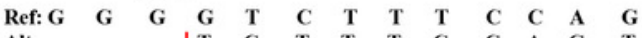
\begin{tabular}{l|llllllllll} 
Alt: & T & C & T & T & T & C & C & A & G & T
\end{tabular}

II-1

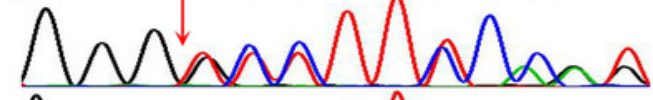

I-5

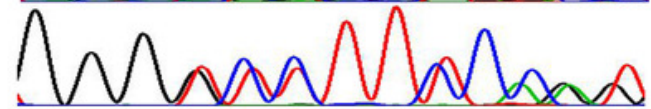

I-6

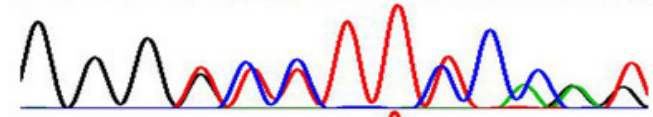

III-1

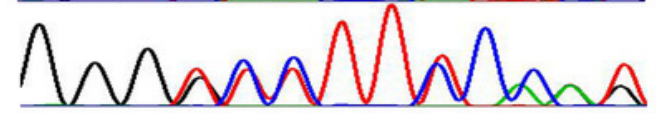

I-2

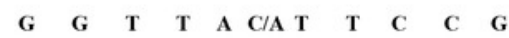

II-1

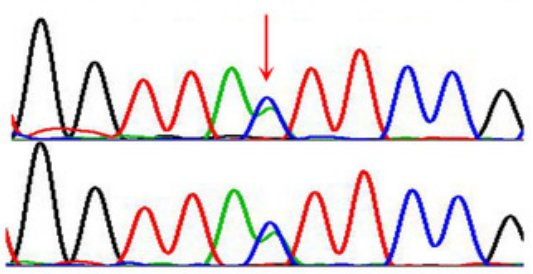

